\title{
Identification of possible non-stationary effects in a new type of vortex furnace
}

\author{
Evgeniy Yu. Shadrin ${ }^{1, *}$, Igor S. Anufriev ${ }^{1}$, and Anatoly P. Papulov ${ }^{1,2}$ \\ ${ }^{1}$ Kutateladze Institute of Thermophysics SB RAS, Novosibirsk, Russia \\ ${ }^{3}$ Novosibirsk State University, Novosibirsk, Russia
}

\begin{abstract}
The article presents the results of an experimental study of pressure and velocity pulsations in the model of improved vortex furnace with distributed air supply and vertically oriented nozzles of the secondary blast. Investigation of aerodynamic characteristics of a swirling flow with different regime parameters was conducted in an isothermal laboratory model (in 1:25 scale) of vortex furnace using laser Doppler measuring system and pressure pulsations analyzer. The obtained results have revealed a number of features of the flow structure, and the spectral analysis of pressure and velocity pulsations allows to speak about the absence of large-scale unsteady vortical structures in the studied design.
\end{abstract}

\section{Introduction}

Currently the most important source for producing electrical and thermal energy is coal combustion. One of the promising technologies is the pulverized coal combustion in a vortex flow. The flow swirling allows solving a number of problems: to increase the residence time of fuel particles in the combustion chamber, which reduces mechanical underburning; to reduce the level of harmful emissions to environmental standards; to optimize the design of boiler equipment; and to ensure effective management of the combustion process. One of the stages in the development of furnaces is the study of their internal aerodynamics in the laboratory models. A detailed study of the main features of the isothermal flow structure allows optimizing the structural and operating parameters of the furnace. It is known that the intensely swirling flows under certain conditions are characterized by the loss in stability of the stationary regime, which may be expressed in the appearance of the precessing vortex core (PVC). Intensive pressure pulsations associated with the PVC cause wear of power plants and deterioration of the performance of vortex devices and have a negative impact on the furnace processes. Therefore, to improve the efficiency and reliability of operation of the vortex combustion devices under development, one must examine possible unsteady effects that occur in the working areas.

In previous works $[1,2]$ authors visualized the vortex structure of the flow in the model of the improved vortex furnace with distributed fuel-air mixture supply and vertically oriented nozzles of the secondary blast. The obtained results were based on measuring the time-averaged flow characteristics and did not allow making an unambiguous conclusion about the vortex core dynamics. The aim of this work is to experimentally study the pulsation characteristics of the flow in this model of the vortex furnace. 


\section{Experimental setup and measurement techniques}

The study of the flow structure and its pulsation characteristics was performed on an automated experimental stand. The scheme of the experimental setup with the mounted three-component (3D-) LDA system is presented on figure 1-a. The main elements of this setup are: the supply line of compressed air with control and regulating devices; the model of an improved vortex furnace; fog generator (for seeding the investigated flow with tracerparticles); measure devices and computer with specialized software. Air flows through the supply line is injected in the isothermal model of the vortex furnace. The study of this flow is based on a variety of modern optical methods.

The main elements of the vortex furnace are (Fig. 1-b): the combustion chamber; a diffuser; and a cooling chamber ending with a horizontal flue. Dimensions of the model are $\mathrm{XYZ} 320 \times 1200 \times 256 \mathrm{~mm}$ (scale 1:25), and the diameter of the vortex combustion chamber is $320 \mathrm{~mm}$. A distinctive feature of this design compared to the previously studied one [3] (where the additional tangential inlet is located in the lower part of the combustion chamber), is the vertical arrangement of the nozzles of the secondary blast, and the presence of the "visor" inside the vortex chamber to prevent entrainment of fuel particles from the combustion chamber. The advantages of this design of the vortex furnace are described in $[1,2]$.

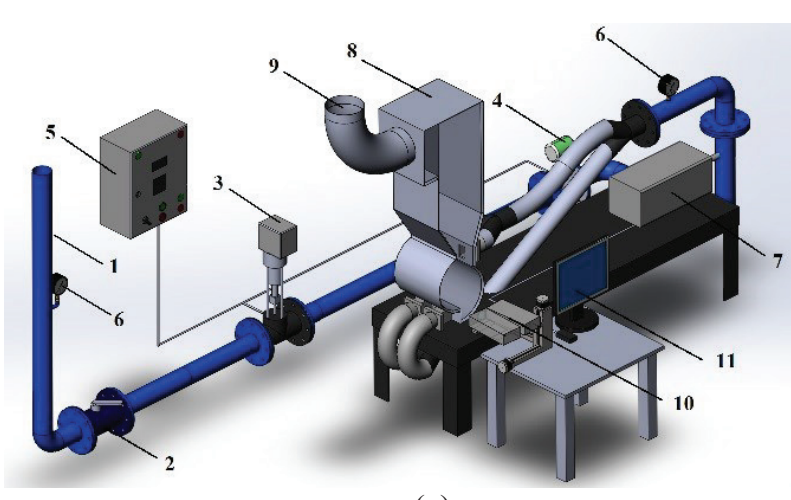

(a)

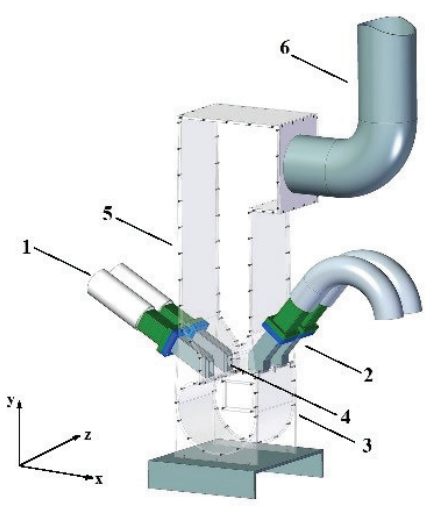

(b)

Fig. 1. (a) Experimental stand scheme: 1 - supply line of compressed air, 2 - stop valve, 3 - shut-off control valve, 4 - flow converter, 5 - control cabinet, 6 - manometer, 7 - fog generator, 8 - vortex furnace model, 9 - ventilation, 10 -measuring system, 11 - computer. (b) Laboratory model of the improved vortex furnace: 1 - main nozzles, 2 - secondary nozzle, 3 - combustion chamber, 4 diffuser, 5 - cooling chamber, 6 - flue.

Measurements of pressure fluctuations were carried out using noise analyzer Bruel\&Kjaer (pressure measurement up to $103.5 \mathrm{kPa}$, frequency measurement $-4.2 \mathrm{~Hz} \div 20$ $\mathrm{kHz}$, sensitivity - $54.9 \mathrm{mV} / \mathrm{PA})$. The sensor was placed inside the vortex combustion chamber with a metal sampler - thin-walled tube with a diameter of $2.2 \mathrm{~mm}$ and a length of $160 \mathrm{~mm}$. The transfer function of the sampler is presented in [4], which shows its applicability without adjusting to frequencies of $\sim 100 \mathrm{~Hz}$. The measurements were carried out close to the conventional center of the vortex chamber $(x=y=160 \mathrm{~mm}, z=126 \mathrm{~mm})$ at various operating parameters $(\gamma=1 \div 4$, where $\gamma$ is the ratio of the flow rates through the main and additional nozzles). The signal digitized by the ADC (L-CARD E14-440), was expanded into a spectrum using fast Fourier transform.

To diagnose the flow velocity pulsations the two-component (2D-) laser Doppler anemometer LAD-05 (frequency up to $3 \mathrm{kHz}$ ), developed at IT SB RAS was used. The method of laser Doppler anemometry is based on measuring the displacement velocity of 
the particles (tracers) suspended in the flow. The measurements were performed in the range of Reynolds numbers $3 \cdot 10^{5}<\operatorname{Re}<6 \cdot 10^{5}$, calculated on the diameter of the vortex chamber $(320 \mathrm{~mm})$ and the velocity module in the top burners $\left(V_{0}=5 \div 25 \mathrm{~m} / \mathrm{s}\right)$. These conditions $\left(\operatorname{Re}>10^{4}\right)$ provide the self-similarity regime and the applicability of the results of physical modeling to the analysis of the structure of isothermal flow in a full-size furnace. The measurements were carried out in a period of time (about $1 \mathrm{~min}$ ) sufficient to obtain 16384 measurements for each component $\left(2^{14}\right)$ to facilitate further processing using the fast Fourier transform (FFT).

\section{Measurement results and their analysis}

Earlier, based on independent measurement techniques, using 3D-LDA [1] and Stereo PIV (Particle Image Velocimetry) systems [2], the authors studied the aerodynamics of a model of the studied vortex furnace with distributed tangential air supply. Based on the "minimum total pressure criterion" [5] the vortex flow structure was visualized. Figure 2-a presents the vector velocity field (a section in the "center of the nozzle"), obtained by PIVmeasurements [1] as well as the isosurface of dynamic pressure $\left(p_{\mathrm{dyn}}=0.25 \mathrm{~Pa}\right.$, Fig 2-b), visualizing the vortex flow structure [2]. It has a W-shaped form, which is typical for the well-known vortex furnace of CBTI [6]. For comparison, the figure shows the isosurface of total pressure and the plotted distribution of the Q-criterion (Fig. 2-c), obtained by numerical simulation [7].

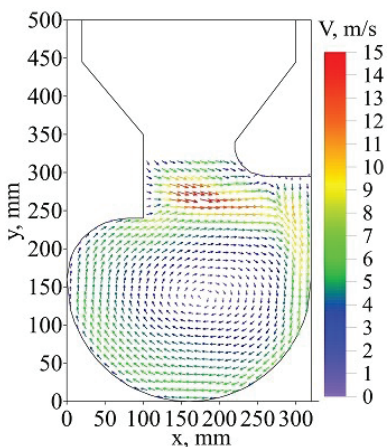

(a)

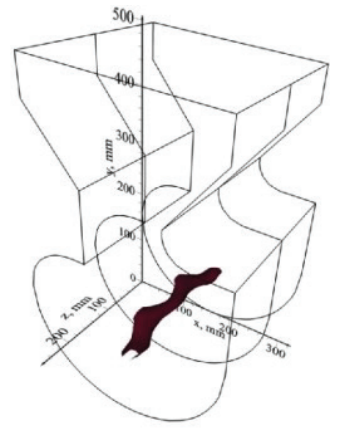

(b)

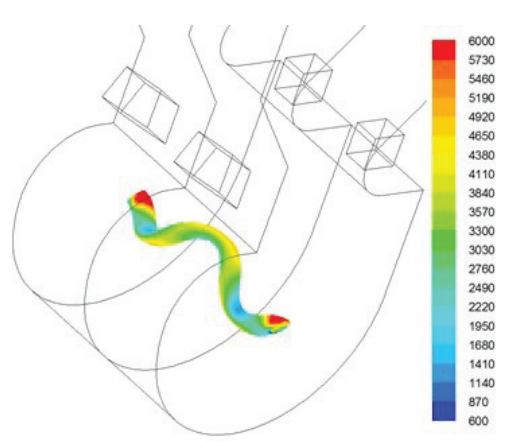

(c)

Fig. 2. The vector velocity field in the cross-section "over the nozzle center" (a); isosurface of dynamic pressure (b); isosurface of total pressure (c).

To analyze stationarity of the revealed vortex structure the pressure fluctuations were measured in the central part of combustion chamber. Spectra for the microphone signal (U, Volts) obtained for different ratios of flow rates through the main and additional nozzles $(\gamma)$ are presented in figure 3. They have a complex form with multiple peaks. However, the position of these peaks neither depends on the flow rate ratio, nor on the magnitude of the total flow, which indicates the absence of the unsteady vortex structures (such as precession vortex core or other structures [8]). The presented peaks characterize the model as an acoustic resonator [6]. 

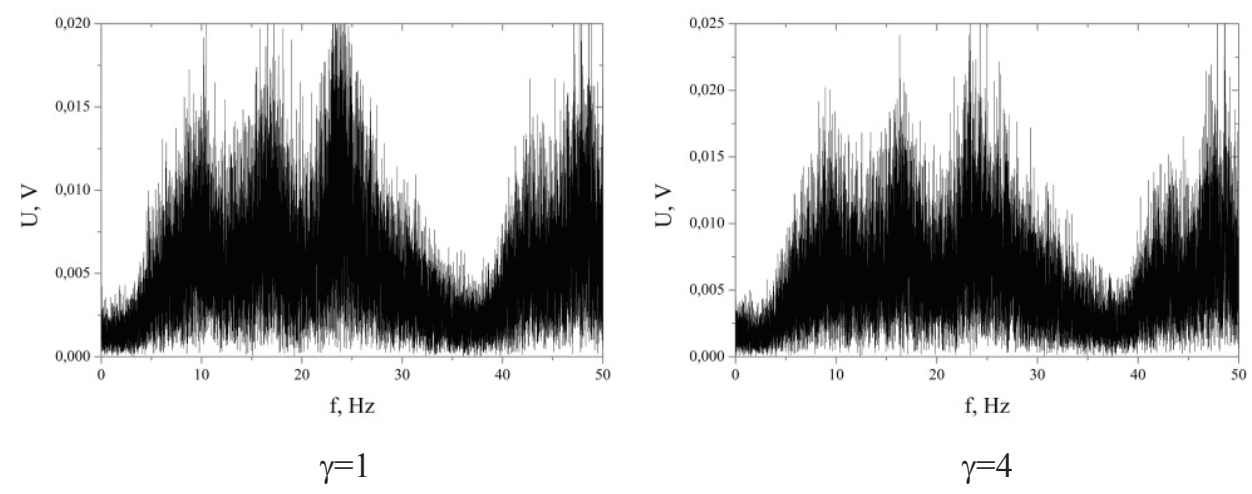

Fig. 3. Spectra of pressure pulsations in the center of the combustion chamber at different ratios of the flow rates through the primary and secondary nozzles $(\gamma)$.

The noise analyzer used in the measurements is limited in frequency range. Therefore, the analysis of pulsations at low frequencies $(<5 \mathrm{~Hz})$ was performed with the use of the laser Doppler anemometer LAD-05. The measurements were carried out at different points of the model in the vicinity of the conventional axis of the curved vortex core and near the entrance nozzles, for different values of initial velocity. Figure 4 shows typical spectra of pulsations of the horizontal velocity component (normalized on the initial velocity $\mathrm{V}_{0}$ ) in the point $(x=150 \mathrm{~mm} ; \mathrm{y}=100 \mathrm{~mm}, \mathrm{z}=64 \mathrm{~mm}$ ) in a plane passing through the centers of the nozzles. The complete absence of peaks confirms the earlier conclusion about the PVC absence. These spectra are characteristic for all points of measurements.
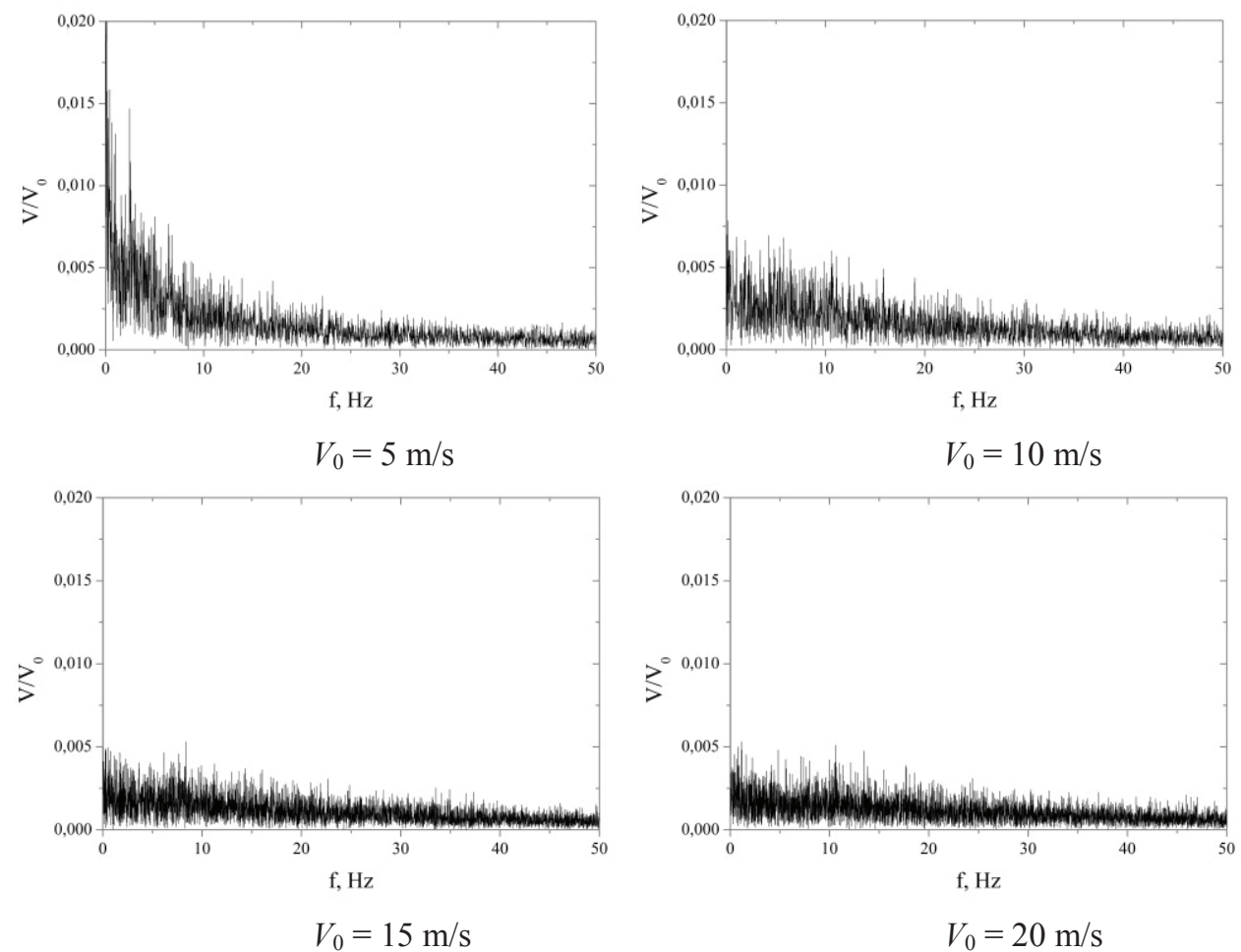

Fig. 4. Pulsation spectra of the $x$ - velocity component for different values of initial velocity $(\gamma=1)$. 


\section{Conclusions}

Using the modern methods of measurements the pulsation characteristics of the swirling flow have been investigated in the improved model of the vortex furnace with distributed fuel-air supply and vertically oriented nozzles of the secondary blast. The results of the spectral analysis of pulsations of pressure and velocity of the turbulent swirling flow with different prove the stability of the stationary structure of the vortex core flow in the studied improved model of the vortex furnace. The absence of negative effects associated with the vortex core precession is one of the important practical advantages [9] of the studied furnace design.

Research was supported by the Russian Science Foundation (Project No. 14-19-00137).

\section{References}

1. Anufriev I. S., Strizhak P. A., Chernetskii M. Yu., Shadrin E. Yu., Sharypov O. V., Tech. Phys. Letters 41, 727 (2015)

2. Anufriev I. S., Kuibin P. A., Shadrin E. Yu., Sharaborin D. K., Sharypov O. V., Thermophys. Aeromech. 6, 621 (2016)

3. Krasinsky D. V., Salomatov V. V., Anufriev I. S., Sharypov O. V., Shadrin E. Yu., Anikin Yu. A., Thermal Engineering 62, 117 (2015)

4. Litvinov I.V., Shtork S.I., Kuibin P.A., Alekseenko S.V., Hanjalic K., Int. J. Heat and Fluid Flow 42, 251 (2013)

5. Dubief Y, Delcayre F., Journal of Turbulence 1, 22 (2000)

6. Anikin Yu. A., Anufriev I. S., Shadrin E. Yu., Sharypov O. V., Thermophys. Aeromech. 21, 775 (2014)

7. Krasinsky D. V., Sharypov O. V., Journal of Engineering Thermophysics 24, 348 (2015)

8. Okulov V. L., Naumov I. V., Sorensen J. N., Tech. Phys. 52, 583 (2007)

9. Chernetskiy M.Yu., Dekterev A.A., Burdukov A.P., Hanjalić K., Fuel. 135, 443 (2014) 